\title{
Modeling collaborative learning: case of clinical reasoning
}

\author{
Type of article: Conference abstract
}

\author{
Mohamed Abderraouf Ferradji ${ }^{1}$, Djalal Hedjazi ${ }^{2}$ \\ ${ }^{1}$ Ph.D Student, LaSTIC Laboratory, Department of computer science, Faculty of \\ Maths \& Computing, University of Batna 2, Batna, Algeria \\ 2. Assistant professor, LaSTIC Laboratory, Department of computer science, \\ Faculty of Maths \& Computing, University of Batna 2, Batna, Algeria \\ ferradji.abderraouf@gmail.com
}

\begin{abstract}
:
Background: Collaborative learning is an important pedagogical strategy which gained a huge interest in critical domains such as the medical field. However, to ensure the quality of this learning method, it is necessary to focus intention not only on the cognitive aspect but also on the social activities that represent an essential issue during collaborative learning sessions. Our objective in this study is to highlight the collaborative aspect in the group learning method of clinical reasoning.

Methods: In this work, we have focused on cognitive studies that are interested in the clinical reasoning processes, while proposing a model dedicated to the design of collaborative clinical reasoning learning environment in synchronous mode. This model is primarily interested in social activities that have a strong influence on the collaborative learning effectiveness, and they are generally treated implicitly by basing on the improvisation and spontaneity of the learners group.

Results: The research idea was embodied through a collaborative learning clinical reasoning environment based on Web 2.0 technologies. We chose this technology to benefit from its ease of use and from its technical performances that can significantly contribute to the development of the cognitive and social aspects in the collaborative learning environment.

Conclusion: Our first contact with medical students showed us that they are appreciating this learning method. Indeed, to evaluate objectively our choices reliability, we plan to accomplish this research with a quantitative study based on real experiences with clinicians and medical students. The suggested study will allow us to collect the necessary lessons to work in depth on the relevant questions concerning the cognitive and social activities in the collaborative clinical reasoning learning.
\end{abstract}

Keywords: Clinical reasoning, Medical education, Collaborative learning, Social knowledge building, Synchronous/Asynchronous interaction

\section{Declaration of conflicts}

This article is selected from the abstract's book of the International Conference on Health Sciences and Medical Technologies,10-12 October 2017, Tlemcen, Algeria, ICHSMT' 17.

\section{Authors' biography}

No biography 
Medical Technologies Journal, Volume: 1, Issue: 3, July -September 2017, Pages: 52-53. DOI: https://doi.org/10.26415/2572-004X-vol1iss3p52-53

\section{References}

No references 